\title{
El aula invertida en el aprendizaje de los estudiantes: revisión sistemática
}

\section{The flipped classroom in student learning: a systematic review}

Silvia Ysela Cabrera Larreategui ${ }^{1}$; scabreral@ucvvirtual.edu.pe

Emérita Mercedes Rojas Yalta1; erojasya@ucvvirtual.edu.pe

Oscar López Regalado1; Iregaladoo@ucvvirtual.edu.pe

D Domel Montenegro Torres²; domoto128@gmail.com

\section{Resumen}

En el ámbito educativo, los videos son utilizados como medio didáctico para generar aprendizaje; el objetivo es analizar el impacto de la producción científica del aula invertida en el aprendizaje de los estudiantes entre el 2018 - 2021. Es una revisión sistemática de carácter bibliométrico, usa operadores booleanos y diagrama prisma, obteniendo como muestra 22 artículos publicados en revistas indexadas a Scopus, ESBCO, Academic OneFile y ERIC. Los resultados permiten afirmar que tanto varones como mujeres realizan publicaciones en igual proporción; los años con más publicaciones son el 2020 y 2019, la mayoría de publicaciones en cuanto a la metodología se inclina por el enfoque cuantitativo de tipo descriptivo, con diseño cuasi experimental. España es el país que lleva la delantera en publicación. Se concluye que el aula invertida influye positivamente en el aprendizaje de los estudiantes y su implementación va a depender del grado de motivación y compromiso que asuman los involucrados.

Palabras clave: video interactivo, aula invertida, aprendizaje semipresencial, estudiante, revisión sistemática.

\begin{abstract}
In the educational field, videos are used as a didactic resource to generate learning; the objective of the article was to analyze the impact of the scientific production about the use of the flipped classroom model on student learning between 2018 - 2021. This is a systematic bibliometric review, using Boolean operators and the prism diagram. The sample was 22 articles published in journals indexed to Scopus, ESBCO, Academic OneFile and ERIC. Based on the results, it can be stated that both men and women publish publications in the same proportion; 2020 and 2019 are the years with the most publications; the descriptive and quasi-experimental designs, on the quantitative approach, were more frequently used. Spain is the country that takes the lead in publication. Finally, it is concluded that the flipped classroom positively influences on student learning and its implementation will depend on the degree of motivation and commitment assumed by those involved.
\end{abstract}

Keywords: interactive video, flipped classroom, blended learning, student, systematic review.

${ }^{1}$ Universidad César Vallejo (Perú)

${ }^{2}$ Institución Educativa Superior Tecnológica Pública "Utcubamba" (Perú) 


\section{INTRODUCCIÓN}

El estudiante al ser un nativo digital tiene que desarrollar sus habilidades en torno a las necesidades de aprendizaje que nos ofrece el mundo actual, surgiendo "El aula invertida en el aprendizaje de los estudiantes" como una alternativa para formar estudiantes competentes, que se desempeñen de manera proactiva en la sociedad.

La aplicación de la metodología del aula invertida (Flipped Classroom), viene desde hace muchos años atrás, en diferentes áreas de la enseñanza. Bárbara Walvoord y Virginia Johnson, en el año 1998 ya recomendaban la aplicación de este modelo sin el uso indispensable de la tecnología (Keengwe \& Onchwari, 2015); sin embargo, los que desempeñaron un papel importante y concluyente fueron en el año 2007, Jonathan Bergman y Aarom Sams, profesores universitarios que, ayudaron a alumnos que no habían podido asistir a clase, a través de presentaciones en Power Point, distribuidas en Internet con acogida por muchos más (Bergmann, 2012; Garzón \& Ibañez, 2020).

El aula invertida y el aprendizaje tienen su fundamento teórico en el enfoque positivista, se cimienta en el paradigma constructivista, que señala que las únicas herramientas para lograr aprendizajes significativos son nuestros sentidos, por lo tanto, se afirma que el conocimiento, no puede ser transferido de un cerebro a otro de manera intacta (Carretero, 1993, como se citó en Sánchez, 2018). Asimismo, los representantes del constructivismo dan a conocer que a partir de la manipulación de las cosas se adquiere un aprendizaje duradero. También, el conectivismo considerado como la nueva teoría de la era digital de George Siemens, brinda un vistazo a las habilidades de aprendizaje y las acciones indispensables para que los estudiantes prosperen en una era digital (Gutierrez, 2012); también, se aborda al aprendizaje desde la perspectiva de la tecnología; se evidencia al conectivismo como una red interconectada, que determina el nivel de aprendizaje que da realce al aprender haciendo, tomando al error como la clave para aprender (Peña, 2020).

Por ello la metodología del aula invertida, es considerada como una forma distinta de aprender, en la que los estudiantes obtienen nuevos saberes no solo en la escuela sino en otros espacios como su casa; por medio de videos, audios educativos, imágenes y a través de plataformas virtuales asequibles al estudiante (Prieto et al., 2016). Se conceptualiza también, como la inversión de roles desempeñados en el aula, por ello, el profesor toma el papel secundario como orientador del aprendizaje y el estudiante adquiere el rol protagónico, aprendiendo con autonomía en un ambiente que no solo es el aula (Basso et al., 2018; Fonseca \& Melo, 2019). Visto al aprendizaje, como el proceso que nos lleva al cambio, a través de la experiencia misma en interacción con el medio que lo rodea, brindando sentido a lo que hacemos (Medina et al., 2019; Vigueras \& Espinar, 2020). En esta indagación se toma la dimensión estudiante, como la persona que está siguiendo estudios educativos en beneficio de sí mismo y de la sociedad, considerándolo como el sujeto con capacidad para procesar información de diversa índole, pero que presenta cierta limitación en algunos aspectos y necesita ser acompañado, guiado y orientado (García et al., 2018; Ruiz \& Torres, 2020). 
La importancia de esta investigación radica en presentar información relevante, de interés para el investigador; ante ello surge la siguiente interrogante: ¿Cuál es el aporte teórico, año de publicación, país y género de autores de los artículos científicos relacionados con el aula invertida y el aprendizaje de los estudiantes durante el periodo 2018 al 2021 ? Respuesta que está fundamentada en la revisión sistemática de material bibliográfico con rigor científico.

El objetivo de esta investigación es analizar el aporte teórico, año de publicación, país y género de autores de los artículos científicos relacionados con el aula invertida y el aprendizaje de los estudiantes durante el periodo 2018 al 2021. La búsqueda de información se ha realizado en las bases de datos Scopus, ESBCO, Academic OneFile y ERIC.

\section{MÉTODOS}

Con el propósito de cumplir con el objetivo propuesto, se ha seguido la revisión sistemática de la información, con un enfoque cuantitativo - retrospectivo (Hernandez et al., 2014). El diseño de esta investigación es bibliométrico (Solano et al., 2009). La rigurosidad y la calidad de la revisión sistemática se basa en el cumplimiento de reglas estandarizadas para este tipo de investigaciones (Kitchenham \& Charters, 2007; Ramírez, et. al. 2018, como se citó en Hinojo et al., 2019).

Para realizar la búsqueda de información con rigor científico, se tuvo en cuenta las bases de datos académicas como Scopus, ESBCO, Academic OneFile y ERIC, seleccionadas porque son las más reconocidas internacionalmente por el índice de impacto que presentan en el área de la investigación; inicialmente se realizó una búsqueda general y avanzada; considerando como periodo de búsqueda el último trimestre del año 2020. La indagación se llevó a cabo por términos de palabras descriptoras en cada una de las bases de datos mencionadas "aula invertida" "aprendizaje" "estudiante" considerando sus sinónimos "aprendizaje invertido, video interactivo, flipped classroom, aprendizaje semipresencial y aprendiz". Se utilizó los operadores booleanos "AND" $y$ "OR" que nos muestran resultados que contengan al menos uno de los dos términos (Gutiérrez \& Serrano, 2018; López, 2020).

En referencia a los criterios de inclusión se consideró: artículos científicos publicados entre los años 2018 al 2021, escritos en español, publicados en revistas indexadas a Scopus, ESBCO, Academic OneFile y ERIC, todos de acceso abierto disponibles para descargar. En cuanto a la revisión a texto completo se ha considerado los criterios de inclusión, utilizando la herramienta CEBMa, que brinda soporte para tomar decisiones acertadas con amplitud, profundidad y exhaustividad en la búsqueda; verificando que los artículos seleccionados como muestra, cumplan con el 50\% más uno de los criterios propuestos (Barends et al., 2017). Se han excluido aquellos artículos que no son científicos, libros, ensayos, páginas Web; también los que están publicados en otro idioma que no sea el español, los documentos no disponibles para descargar y artículos que no se centran en las palabras claves.

Asimismo, se ha utilizado el diagrama de flujo PRISMA para revisiones sistemáticas, herramienta diseñada con el fin de perfeccionar y clarificar la publicación de revisiones 
sistemáticas (Urrútia \& Bonfill, 2010). Obteniendo una muestra de estudio de 22 artículos científicos, considerando como variables de estudio para esta investigación el año de publicación, género, país y aporte teórico, se considera la temporalidad de 2018 al 2021 porque en los últimos años esta metodología se viene implementando con mayor frecuencia en la educación.

El análisis bibliométrico de la información recopilada entre los años 2018 y 2021 está basada en los criterios de búsqueda para la localización, recuperación y procesamiento de esta información, accediendo a diversas bases de datos.

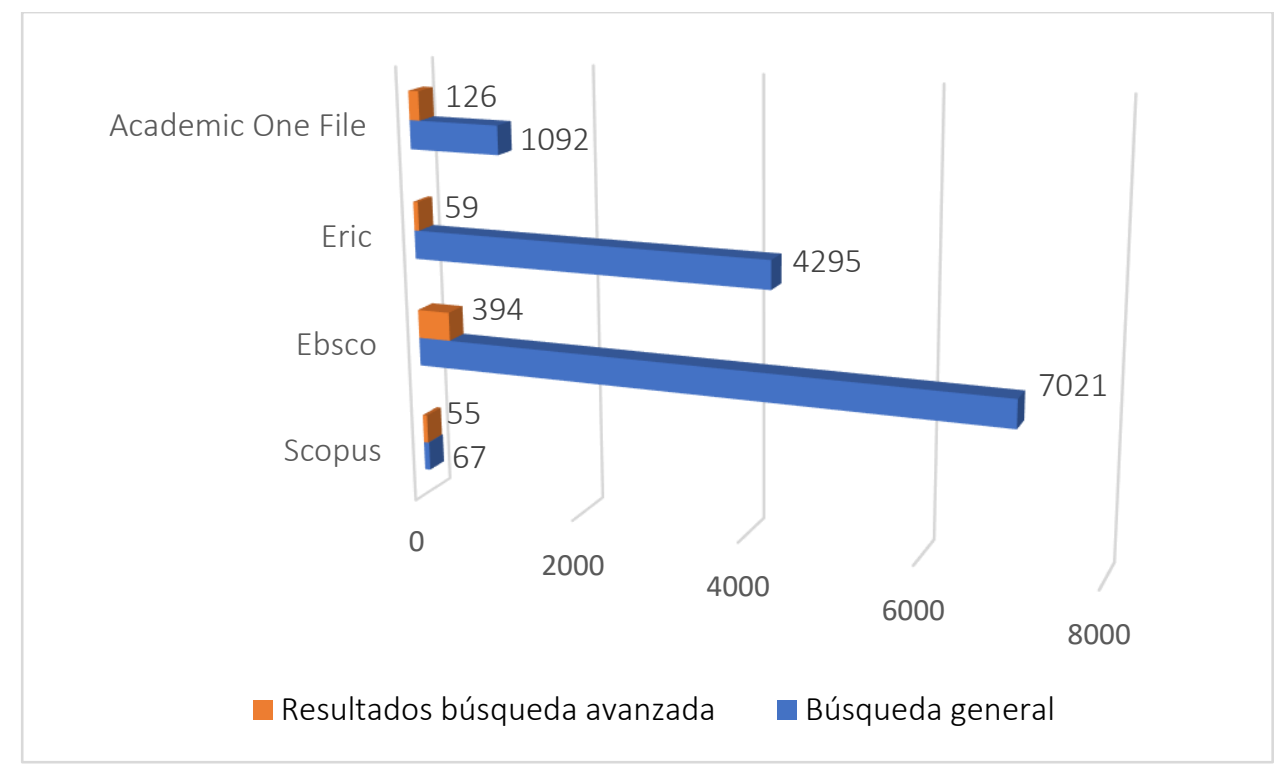

Figura 1. Búsqueda de fuentes de información

Los artículos analizados corresponden a las bases de datos, Scopus, ESBCO, Academic OneFile y ERIC, (Error! Reference source not found.). Se realizó la búsqueda general por nombre de la variable obteniendo 12475 documentos y en búsqueda avanzada por términos de palabras descriptoras - sinónimos en cada una de las bases de datos mencionadas un total de 634 artículos científicos. Ante ello se determinó que la mayor cantidad de artículos se encuentran en la base de datos EBSCO con 394, y en menor proporción están en Scopus con 55 publicaciones. 


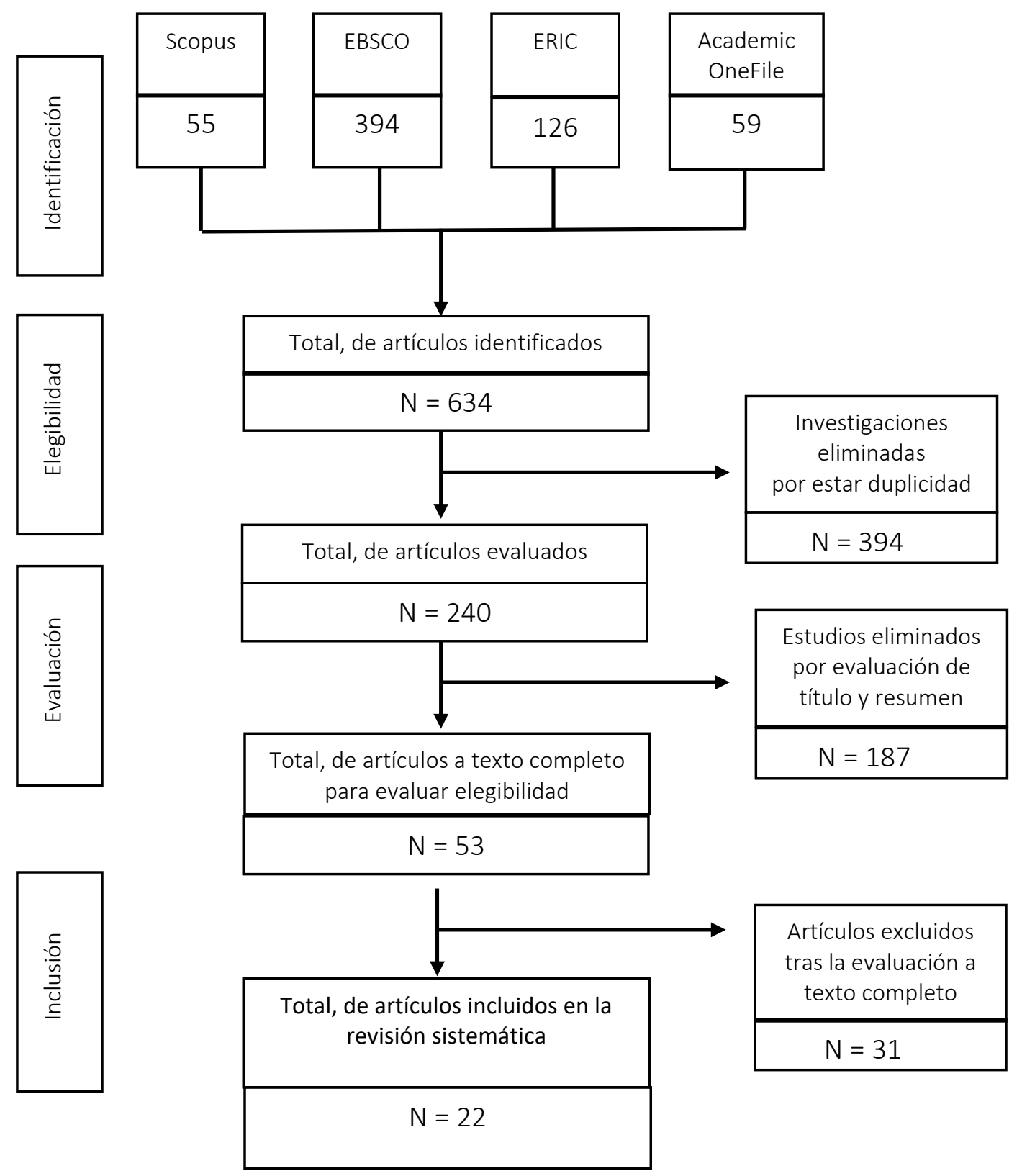

Figura 2. Diagrama de flujo Prisma de inclusión e exclusión

Con el diagrama PRISMA, se garantizó la legitimidad de la información con rigor científico, del total de artículos considerados en la identificación ( 
Figura 22); se procedió a realizar un primer filtro, teniendo en cuenta la elegibilidad de la información seleccionada, por ello se eliminó aquellas investigaciones que están duplicadas, concluyendo que hay 394 artículos publicados en más de una base de datos; restándole esta cantidad al total de artículos inicialmente escogidos se obtiene 240 artículos. Luego de ello se procedió a la evaluación, que es el segundo filtro, correspondiendo la eliminación de estudios evaluando el título, palabras claves y resumen, teniendo como resultado un total de 187 artículos; los mismos que al ser restados a la cantidad anterior, se obtiene un total de 53 publicaciones. Para considerar los artículos que serán incluidos en esta investigación se sometió a la revisión a texto completo respondiendo según los criterios establecidos por el Center for Evidence-Based Management, (CEBMa), considerando el 50\% más uno para la inclusión; por lo que, se excluyen 31 artículos, quedándonos con una muestra para realizar el análisis profundo de la información de 22 artículos científicos.

\section{RESULTADOS}

Tabla 1.

Artículos según, país, año de publicación y género de primer autor

\begin{tabular}{|l|c|c|}
\hline \multicolumn{1}{|c|}{ País } & Artículos & $\%$ \\
\hline España & 14 & 63.6 \\
\hline Colombia & 4 & 18.3 \\
\hline Chile & 2 & 9.1 \\
\hline México & 1 & 4.5 \\
\hline Cuba & 1 & 4.5 \\
\hline
\end{tabular}

\begin{tabular}{|c|c|c|}
\hline Año & Artículos & $\%$ \\
\hline 2018 & 5 & 22.7 \\
\hline 2019 & 8 & 36.4 \\
\hline 2020 & 8 & 36.4 \\
\hline 2021 & 1 & 4.5 \\
\hline
\end{tabular}

\begin{tabular}{|c|c|c|}
\hline Género & Artículos & $\%$ \\
\hline Femenino & 11 & 50 \\
\hline Masculino & 11 & 50 \\
\hline
\end{tabular}

Revisando la información presentada (Tabla 1.

Artículos según, país, año de publicación y género de primer autor), se determinó que el 2020 y 2019, son los años donde más publicaciones en idioma castellano se han realizado en revistas indexadas 
a bases de datos de prestigio mundial. Se comprobó que, desde el 2018 hasta enero del 2021, tanto el género femenino como masculino, han publicado la misma cantidad de artículos. En cuanto al país con más publicaciones, España, lleva la delantera con 14 artículos publicados.

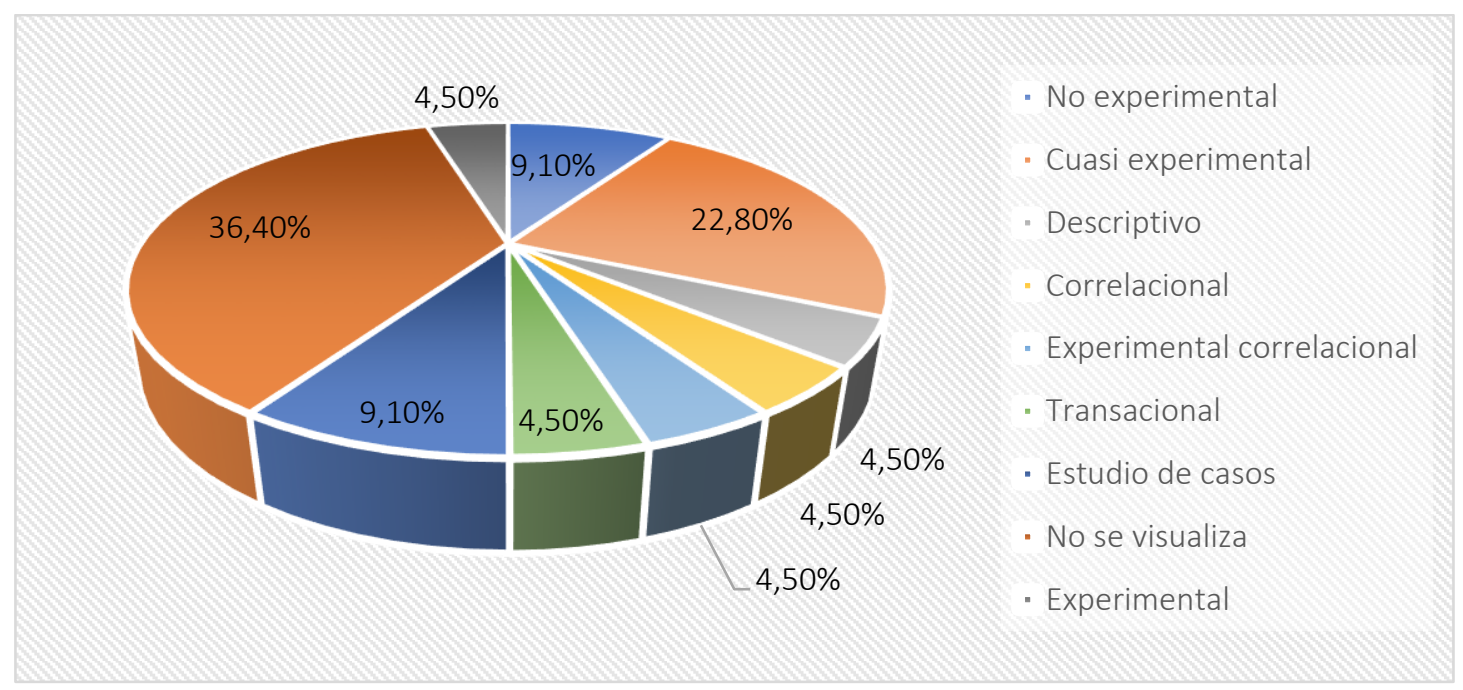

Figura 3: Diseño de investigación

En la (Figura 3) se aprecia que $36.40 \%$ de artículos no contemplan el tipo de diseño de investigación, el $22.80 \%$ son cuasi experimentales, el $9.10 \%$ corresponden al estudio de casos y no experimentales respectivamente y el $4.50 \%$ pertenecen a los demás tipos de diseños.

Tabla 2.

Artículos según aporte al Estudio

\begin{tabular}{|c|l|l|}
\hline Autores & Objetivo de investigación & Conclusiones relevantes \\
\hline $\begin{array}{c}\text { (Cuenca } \\
\text { et al., 2021) }\end{array}$ & $\begin{array}{l}\text { Establecer si la metodología Flipped } \\
\text { Classroom aumenta la motivación del } \\
\text { alumnado universitario y, por ende, el } \\
\text { ambiente de aprendizaje y en } \\
\text { consecuencia la mejora del estado de } \\
\text { Flow. }\end{array}$ & $\begin{array}{l}\text { La metodología Flipped Classroom es por hoy, una } \\
\text { de las metodologías más eficaces para incrementar } \\
\text { la motivación del alumnado, así como su } \\
\text { rendimiento y su estado de Flow. }\end{array}$ \\
\hline $\begin{array}{c}\text { (Garzón \& } \\
\text { Ibañez, }\end{array}$ & $\begin{array}{l}\text { Analizar el logro de aprendizaje en } \\
\text { estudiantes de décimo en un colegio } \\
\text { público en Bogotá, aplicando la la } \\
\text { estrategia de aula invertida. }\end{array}$ & $\begin{array}{l}\text { En el grupo con aula invertida es preciso incorporar } \\
\text { estrategias de control que garanticen que las } \\
\text { puede ser encaminado a que las actividades de la } \\
\text { lección en casa y las de los deberes en clase forman } \\
\text { un conjunto planificado de acciones. }\end{array}$ \\
\hline $\begin{array}{l}\text { (Hernández } \\
\text { et al., 2020) }\end{array}$ & $\begin{array}{l}\text { Determinar el efecto del modelo aula } \\
\text { invertida en el rendimiento académico } \\
\text { ylas opiniones de los estudiantes de un } \\
\text { programa de formación de docentes. }\end{array}$ & $\begin{array}{l}\text { Los estudiantes reconocieron la conveniencia y los } \\
\text { beneficios pedagógicos del enfoque aula invertida. } \\
\text { Permitiendo, reflexionar en dos perspectivas, por } \\
\text { un lado, como estudiantes sobre el compromiso con } \\
\text { su propio aprendizaje y la necesidad de } \\
\text { desarrollarlo de manera grupal y autónoma y como } \\
\text { futuros docentes, sobre la importancia de }\end{array}$ \\
\hline
\end{tabular}


Cabrera, S. Y., Rojas, E. M., Montenegro, D., y López, O.

\begin{tabular}{|c|c|c|}
\hline & & $\begin{array}{l}\text { modernizar las prácticas pedagógicas con modelos } \\
\text { innovadores. }\end{array}$ \\
\hline $\begin{array}{l}\text { (Mengual } \\
\text { et al., 2020) }\end{array}$ & $\begin{array}{l}\text { Conocer la influencia de factores } \\
\text { externos y constitutivos a la } \\
\text { metodología flipped learning sobre los } \\
\text { resultados de aprendizaje obtenidos } \\
\text { por el discente en el proceso de } \\
\text { enseñanza y aprendizaje. }\end{array}$ & $\begin{array}{l}\text { Esta metodología tecnológica es necesario contar } \\
\text { con un ambiente de trabajo idóneo para la } \\
\text { visualización de los contenidos en formato digital, } \\
\text { esto es, contar con los recursos tecnológicos } \\
\text { necesarios, así como un ambiente y espacio de } \\
\text { trabajo adecuado a los requerimientos de una } \\
\text { actividad formativa fuera del aula. }\end{array}$ \\
\hline $\begin{array}{l}\text { (Espada } \\
\text { et al., 2020) }\end{array}$ & $\begin{array}{l}\text { Analizar el rendimiento académico, la } \\
\text { satisfacción y la percepción de la } \\
\text { experiencia educativa de los } \\
\text { estudiantes universitarios hacia el uso } \\
\text { del método de flipped classroom. }\end{array}$ & $\begin{array}{l}\text { El método de clase invertida es adecuado para } \\
\text { implementarlo en el contexto universitario, } \\
\text { permitiendo extrapolar los resultados a otras } \\
\text { disciplinas académicas. Mejora de la adquisición de } \\
\text { los contenidos y la percepción del alumnado en su } \\
\text { rendimiento académico, incrementa la motivación } \\
\text { hacia el aprendizaje, favorece las interacciones } \\
\text { sociales con los compañeros, mejora la percepción } \\
\text { del rol del docente en el aula como guía en el } \\
\text { aprendizaje. }\end{array}$ \\
\hline $\begin{array}{l}\text { (Guillén } \\
\text { et al., 2020) }\end{array}$ & $\begin{array}{l}\text { Conocer la eficacia de la metodología } \\
\text { Flipped Classroom apoyado por la } \\
\text { plataforma virtual Blackboard sobre las } \\
\text { actitudes hacia la estadística del } \\
\text { alumnado del Grado de educación } \\
\text { Primaria. }\end{array}$ & $\begin{array}{l}\text { Apostar por metodologías como el Flipped } \\
\text { Classroom en un contexto formativo e-learning ha } \\
\text { mejorado las actitudes del alumnado respecto al } \\
\text { aprendizaje de la Estadística, donde el alumnado } \\
\text { que siguió la asignatura en e-learning redujo su nivel } \\
\text { de ansiedad y mejoró la percepción de utilidad hacia } \\
\text { esta materia. }\end{array}$ \\
\hline $\begin{array}{l}\text { (Mendoza } \\
\text { \& Ledo, } \\
\text { 2020) }\end{array}$ & $\begin{array}{l}\text { Fortalecer la metodología del aula } \\
\text { invertida a través de trabajo grupal } \\
\text { denominado mesas invertidas de } \\
\text { aprendizajes, para incidir en el } \\
\text { mejoramiento del proceso enseñanza } \\
\text { aprendizaje. }\end{array}$ & $\begin{array}{l}\text { Esta herramienta didáctica por ser novedosa y } \\
\text { adaptable a cualquier medio educativo, se propone } \\
\text { utilizarla como técnica para fortalecer el trabajo } \\
\text { grupal de forma cooperativa, participativa, } \\
\text { contextualizada, interdisciplinar e intercultural. }\end{array}$ \\
\hline $\begin{array}{l}\text { (Domínguez } \\
\qquad \& \\
\text { Palomares, } \\
\text { 2020) }\end{array}$ & $\begin{array}{l}\text { Realizar un pilotaje en la asignatura } \\
\text { Tendencias Contemporáneas de la } \\
\text { Educación del Grado de Maestro de } \\
\text { Educación Primaria de la Universidad de } \\
\text { Castilla-La Mancha para evaluar el } \\
\text { grado de satisfacción del alumnado } \\
\text { sobre la metodología "flipped } \\
\text { classroom" }\end{array}$ & $\begin{array}{l}\text { La metodología del "flipped classroom" o "aula } \\
\text { invertida" es útil para el alumnado, respeta su ritmo } \\
\text { de aprendizaje y despierta su motivación por el } \\
\text { estudio. }\end{array}$ \\
\hline $\begin{array}{l}\text { (Sánchez \& } \\
\text { Sánchez, } \\
\text { 2020) }\end{array}$ & $\begin{array}{l}\text { Obtener información que evidencie que } \\
\text { utilizando el modelo flipped classroom } \\
\text { se consigue fomentar la } \\
\text { autorregulación y meta cognición en el } \\
\text { proceso de enseñanza-aprendizaje de la } \\
\text { Didáctica de la Estadística. }\end{array}$ & $\begin{array}{l}\text { El modelo flipped classroom, se ajusta en gran } \\
\text { medida a los deseos de una enseñanza más activa, } \\
\text { participativa, colaborativa, que prepara a las } \\
\text { personas para un mañana impreciso e indefinido, } \\
\text { que fomenta la autonomía y la autorregulación del } \\
\text { aprendizaje. }\end{array}$ \\
\hline $\begin{array}{l}\text { (López } \\
\text { et al., 2019) }\end{array}$ & $\begin{array}{l}\text { Conocer si el nivel de destrezas } \\
\text { alcanzado en cada una de las } \\
\text { dimensiones que componen el área } 3 \\
\text { de la competencia digital presenta }\end{array}$ & $\begin{array}{l}\text { El cuerpo docente debe concienciarse y hacer un } \\
\text { esfuerzo por actualizar las técnicas, así como los } \\
\text { recursos y enfoques metodológicos empleados en } \\
\text { la formación de sus alumnos, con la finalidad de }\end{array}$ \\
\hline
\end{tabular}


Cabrera, S. Y., Rojas, E. M., Montenegro, D., y López, O.

\begin{tabular}{|c|c|c|}
\hline & $\begin{array}{l}\text { alguna influencia en el uso del flipped } \\
\text { learning como metodología de } \\
\text { enseñanza aprendizaje. }\end{array}$ & $\begin{array}{l}\text { ofrecer un servicio de calidad y adecuado a una era } \\
\text { digital. }\end{array}$ \\
\hline $\begin{array}{l}\text { (Veytia } \\
\text { et al., 2019) }\end{array}$ & $\begin{array}{l}\text { Presentar los resultados de la aplicación } \\
\text { de la clase invertida en la asignatura de } \\
\text { Informática 1, en un grupo de } \\
\text { estudiantado de la Escuela Preparatoria } \\
\text { No. } 1 \text { de una institución pública del } \\
\text { Estado de Hidalgo, y valorar después de } \\
\text { su implementación las fortalezas y } \\
\text { áreas de oportunidad en el desarrollo } \\
\text { de la competencia uso de la tecnología. }\end{array}$ & $\begin{array}{l}\text { El método de clase invertida resultó ser una } \\
\text { transformación con resultados favorables para } \\
\text { atender y combatir a las problemáticas identificadas } \\
\text { en el entorno educativo. Se logró atraer la atención } \\
\text { de los estudiantes con la grabación de videos por la } \\
\text { docente con contenido teórico y práctico, para una } \\
\text { mejor comprensión de los temas. }\end{array}$ \\
\hline $\begin{array}{l}\text { (Sánchez } \\
\text { et al., 2019) }\end{array}$ & $\begin{array}{l}\text { Descubrir la percepción de nuestro } \\
\text { alumnado sobre las potencialidades } \\
\text { pedagógicas que diversos estudios } \\
\text { atribuyen al modelo pedagógico de } \\
\text { clase invertida frente al modelo } \\
\text { pedagógico empleado con más } \\
\text { frecuencia para el mismo núcleo } \\
\text { temático y asignatura de la Facultad de } \\
\text { Ciencias de la Educación de nuestra } \\
\text { universidad. }\end{array}$ & $\begin{array}{l}\text { La didáctica de la clase invertida mediada por } \\
\text { tecnología móvil ha mejorado la percepción de } \\
\text { nuestro alumnado acerca de su propio } \\
\text { aprovechamiento del tiempo de clase y ha } \\
\text { mejorado su motivación hacia las sesiones de } \\
\text { formación universitaria. }\end{array}$ \\
\hline $\begin{array}{l}\text { (Ojando } \\
\text { et al., 2019) }\end{array}$ & $\begin{array}{l}\text { Evaluar una propuesta de formación en } \\
\text { Flipped Classroom dirigida al } \\
\text { profesorado universitario de diferentes } \\
\text { estudios de la Universidad Pontificia } \\
\text { Comillas de Madrid según la percepción } \\
\text { de los participantes y datos recogidos a } \\
\text { partir de la implementación en sus } \\
\text { clases. }\end{array}$ & $\begin{array}{l}\text { El ritmo del cambio metodológico en la enseñanza } \\
\text { superior con soporte TIC es lento y de naturaleza } \\
\text { adaptativa. No es una cuestión meramente } \\
\text { tecnológica. El profesorado universitario necesita } \\
\text { espacios y tiempos para innovar } \\
\text { metodológicamente. }\end{array}$ \\
\hline $\begin{array}{l}\text { (Gaviria } \\
\text { et al., 2019) }\end{array}$ & $\begin{array}{l}\text { Analizar la percepción de estudiantes } \\
\text { universitarios, con respecto a la } \\
\text { utilización de la estrategia pedagogía } \\
\text { aula invertida en los cursos de } \\
\text { formación profesional. }\end{array}$ & $\begin{array}{l}\text { El aula invertida, demanda de una planificación } \\
\text { apoyada en las TICs y articulada con estrategias que } \\
\text { promueven la comprensión, el desarrollo de } \\
\text { competencias y las modalidades grupales como } \\
\text { debates, simposios, foros, seminarios, talleres y } \\
\text { mesas redondas, que permiten que los estudiantes } \\
\text { aprecien el aula invertida como un instrumento } \\
\text { para canalizar y dinamizar su aprendizaje, los } \\
\text { participantes generalmente reaccionaron } \\
\text { positivamente a la mediación en el desarrollo del } \\
\text { curso con videos educativos. }\end{array}$ \\
\hline $\begin{array}{l}\text { (Fúneme, } \\
\text { 2019) }\end{array}$ & $\begin{array}{l}\text { Analizar las implicaciones en el } \\
\text { aprendizaje de los estudiantes del } \\
\text { concepto de la derivada al implementar } \\
\text { el aula invertida, describiendo los } \\
\text { principales factores que benefician o no } \\
\text { el aprendizaje del concepto de derivada } \\
\text { con la incorporación de esta } \\
\text { metodología. }\end{array}$ & $\begin{array}{l}\text { Se puede decir que, si bien esta nueva tendencia en } \\
\text { la enseñanza puede resultar útil en diferentes } \\
\text { aspectos, aún debe ser pensada con mayor } \\
\text { profundidad, de forma que atienda realmente a las } \\
\text { necesidades y dificultades que enfrentan los } \\
\text { estudiantes en sus procesos de aprendizaje. }\end{array}$ \\
\hline
\end{tabular}


Cabrera, S. Y., Rojas, E. M., Montenegro, D., y López, O.

\begin{tabular}{|c|c|c|}
\hline $\begin{array}{c}\text { (García } \\
\text { et al., 2019) }\end{array}$ & $\begin{array}{l}\text { Revisar y planificar la organización de } \\
\text { actividades, replanteando la } \\
\text { metodología curricular utilizada en la } \\
\text { asignatura "Organización Escolar y } \\
\text { Recursos en Educación", de 1o curso del } \\
\text { Grado de Maestro en Educación } \\
\text { Primaria. }\end{array}$ & $\begin{array}{l}\text { Si bien los alumnos consideran positivo el desarrollo } \\
\text { de la asignatura mediante la puesta en práctica de } \\
\text { la metodología Aula Invertida; al mismo tiempo, } \\
\text { indican la necesidad de contar con más tiempo para } \\
\text { la realización de los trabajos, con materiales claros } \\
\text { y bien estructurados e intercalar esta estrategia con } \\
\text { clases magistrales que les permitan afianzar el } \\
\text { aprendizaje de los contenidos tratados en la } \\
\text { materia. }\end{array}$ \\
\hline $\begin{array}{c}\text { (Campillo } \\
\text { et al., 2019) }\end{array}$ & $\begin{array}{l}\text { Identificar el nivel de rendimiento de los } \\
\text { estudiantes en el área de Ciencias } \\
\text { Sociales en la escuela primaria a través } \\
\text { del modelo de aula invertida y su nivel } \\
\text { de motivación de acuerdo con las } \\
\text { percepciones y opiniones de sus } \\
\text { maestros. }\end{array}$ & $\begin{array}{l}\text { La aplicación de este modelo ha demostrado la } \\
\text { funcionalidad para trabajar contenidos de ciencias } \\
\text { sociales de manera más rápida e interactiva, la } \\
\text { flexibilidad para adaptarse a los distintos ritmos de } \\
\text { aprendizaje de cada alumno y el incremento de la } \\
\text { motivación de docentes y discentes. }\end{array}$ \\
\hline $\begin{array}{l}\text { (Gómez \& } \\
\text { Gómez, } \\
\text { 2018) }\end{array}$ & $\begin{array}{l}\text { Rediseñar el plan docente de Teorías de } \\
\text { la Comunicación desde el modelo de } \\
\text { aprendizaje de aula inversa. }\end{array}$ & $\begin{array}{l}\text { El modelo de clase inversa provee las bases para } \\
\text { lograr el objetivo de concebir la aplicabilidad del } \\
\text { conocimiento teórico a la vida profesional del } \\
\text { periodista, gracias a la tutorización eficaz en } \\
\text { actividades de aprendizaje activo y colaborativo de } \\
\text { análisis, síntesis y creación de aprendizajes. }\end{array}$ \\
\hline $\begin{array}{l}\text { (Martínez \& } \\
\text { Esquivel, } \\
\text { 2018) }\end{array}$ & $\begin{array}{l}\text { Evaluar los efectos de un modelo de } \\
\text { instrucción bajo los principios del } \\
\text { aprendizaje invertido en relación al } \\
\text { desempeño lector en inglés de jóvenes } \\
\text { bachilleres, provenientes de un centro } \\
\text { escolar de sostenimiento público en } \\
\text { México. }\end{array}$ & $\begin{array}{l}\text { La aportación de la presente experiencia permite } \\
\text { fortalecer el proceso formativo que considere a la } \\
\text { tecnología idónea, al contexto y al diseño } \\
\text { pedagógico que promueva la labor del estudiante } \\
\text { respetando sus singularidades. }\end{array}$ \\
\hline $\begin{array}{c}\text { (Blasco } \\
\text { et al., 2018) }\end{array}$ & $\begin{array}{l}\text { Conocer la influencia en el aprendizaje } \\
\text { del uso de la metodología Flipped } \\
\text { Classroom, el aprendizaje basado en el } \\
\text { juego y el uso de las redes. }\end{array}$ & $\begin{array}{l}\text { El uso de esta metodología ha resultado beneficioso } \\
\text { para los procesos de enseñanza-aprendizaje y de } \\
\text { interacción y comunicación. En este sentido, parece } \\
\text { que los estudiantes a través de la metodología } \\
\text { Flipped Classroom en combinación con el uso de las } \\
\text { redes sociales disponen de los conocimientos } \\
\text { previos necesarios para resolver las tareas } \\
\text { respetando los ritmos de cada uno de los } \\
\text { estudiantes. }\end{array}$ \\
\hline $\begin{array}{c}\text { (Aguayo } \\
\text { et al., 2018) }\end{array}$ & $\begin{array}{l}\text { Describir la percepción de los } \\
\text { estudiantes acerca de la incorporación } \\
\text { de la metodología flipped classroom en } \\
\text { la asignatura de Inglés Comunicacional } \\
\text { en las carreras de Arquitectura e } \\
\text { Ingeniería Civil Química }\end{array}$ & $\begin{array}{l}\text { Los estudiantes valoraron positivamente la } \\
\text { implementación del aula invertida, pues promueve } \\
\text { el aprendizaje fuera del aula, mediante la } \\
\text { observación de los videos. Perciben que el profesor } \\
\text { tiene un rol de facilitador del aprendizaje, } \\
\text { proporcionando más instancias para desarrollar la } \\
\text { competencia comunicativa. }\end{array}$ \\
\hline $\begin{array}{c}\text { (Basso } \\
\text { et al., 2018) }\end{array}$ & $\begin{array}{l}\text { Presentar una propuesta tecnológica } \\
\text { para la implementación de Flipped } \\
\text { Classroom (FC) o aula invertida como } \\
\text { estrategia metodológica en asignaturas }\end{array}$ & $\begin{array}{l}\text { El modelo T-FliC es una propuesta tecnológica para } \\
\text { la implementación de Flipped Classroom en } \\
\text { asignaturas de distintos niveles de formación en } \\
\text { educación superior, la cual se sustenta en la } \\
\text { incorporación de recursos digitales gratuitos. Se }\end{array}$ \\
\hline
\end{tabular}


de distintos niveles de formación en educación superior. favorece la interacción entre estudiante-estudiante, estudiante-docente y docente-estudiante en el fomento de la metacognición.

En los 22 artículos (Error! Reference source not found.2), se encuentra información interesante que es de beneficio para el investigador que busca difundir el conocimiento, manejo y comprensión de la temática analizada relacionada con la metodología del aula invertida en el aprendizaje de los estudiantes independiente del nivel educativo. Entre los aportes teóricos de la mayoría de los autores se menciona la importancia que tiene la implementación de la metodología del aula invertida en el logro de aprendizajes significativos, se evidencia también, que para incrementar los conocimientos es necesario el involucramiento y compromiso de los estudiantes con su rol protagónico, y los docentes como orientadores y guías en este proceso. Por lo tanto, es necesario tener un manejo intermedio del uso de herramientas tecnológicas, estar motivados y ser perseverantes.

\section{DISCUSIÓN Y CONCLUSIONES}

El total de la muestra fue sometido a un análisis bibliométrico, donde se analizó la información, a partir de la producción de la literatura científica existente (Solano et al., 2009). En referencia al género del primer autor (Error! Reference source not found.1), se determinó que tanto las mujeres como los varones vienen realizando publicaciones en la misma proporción en relación a la variable de estudio; los años 2019 y 2020 presentan la mayor cantidad de investigaciones en idioma español, donde se menciona que es necesario reemplazar la clase tradicional, por nuevas formas de enseñar y aprender (Sandí \& Cruz, 2016). Haciendo una comparación entre los países que más investigaron sobre el tema, España lleva la delantera; en Latinoamérica tenemos a Colombia y Chile, Cuba y México son los países que menos publicaciones tienen en los últimos cuatro años. Se determina que las investigaciones analizadas se realizan en su mayoría en el ámbito académico universitario, proyectándose el aula invertida como una metodología de alto impacto en la educación superior (Hinojo et al., 2019).

Asimismo, se comprobó que la mayoría de artículos revisados especifican el diseño de investigación (Figura 3), de acuerdo a la clasificación establecida (Sepúlveda \& Concepción, 2020), observando estudios descriptivos, cuasi experimentales, correlacionales, estudio de casos y otros, mostrando interés por comprobar empíricamente la estrategia del aula invertida en comparación con las clases tradicionales (Hinojo et al., 2019; Monroy \& Monroy, 2019). Sin embargo existen artículos que no tienen metodología (Basso et al., 2018), esto nos lleva a reflexionar sobre la importancia de la metodología en los trabajos de investigación, a fin de analizar de manera crítica y reflexiva los conceptos y teorías a investigar, ya que nos marca el rumbo a seguir para encontrar resultados exactos y eficaces (Díaz \& Calzadilla, 2016; Hernandez et al., 2014).

En cuanto a los aportes que presentan los investigadores (Error! Reference source not found.2), cabe mencionar que en la mayoría de estudios analizados, los grupos que experimentan esta 
metodología manifiestan un alto porcentaje de aumento en sus calificaciones en comparación con el grupo de clase tradicional (Basso et al., 2018; Gaviria et al., 2019; Veytia et al., 2019); se afirma que el aula invertida es una metodología que mejora el rendimiento de los estudiantes, por ende el aprendizaje es más significativo y duradero; además aumentan la motivación, fortalecen la autonomía, autorregulación, trabajo colaborativo y reduce la ansiedad en los estudiantes (Campillo et al., 2019; Domínguez \& Palomares, 2020; Guillén et al., 2020; Sánchez \& Sánchez, 2020); asimismo hace que los estudiantes se involucren de manera colaborativa, respetando su propia iniciativa. El maestro tiene el rol de implementar el aula invertida, por ello, debe tener conocimiento de herramientas virtuales y estar dispuesto a cambiar la forma de llegar a los estudiantes (López et al., 2019; Ojando et al., 2019). El ambiente de trabajo debe ser idóneo para la visualización de contenidos en formato digital, contar con infraestructura acorde a las disciplinas que se desarrollan, tener iluminación natural, con espacios fuera de ruidos; los factores externos pueden alterar los resultados finales (Mengual et al., 2020). Esta nueva tendencia debe de atender realmente a las necesidades y dificultades que enfrentan los estudiantes en sus procesos de aprendizaje, pues hasta el momento se hace mayor énfasis en cómo enseñar y no en cómo aprender (Fúneme, 2019).

La mayoría de las investigaciones concluyen que la implementación del aula invertida mejora el aprendizaje de los estudiantes, brinda confianza y motivación, fortalece la autonomía, fomenta el trabajo colaborativo, autorregula el aprendizaje, reduce la ansiedad y mejora el rendimiento académico; los docentes deben de apostar por nuevas metodologías que mantengan al estudiante motivado; coincidiendo con la adecuada enseñanza que reciben los estudiantes que tienen menos aptitud para el estudio y una oportunidad de renovación del contenido educativo (Torrecilla \& García, 2020).

Finalmente mencionó como limitaciones de estudio, la búsqueda de información en cuatro bases de datos, restringe la investigación de la gran variedad de literatura que no está indexada en las bases de datos consultadas; el centrarse en el constructo aprendizaje de los estudiantes, impide recoger información sobre los diversos beneficios que trae la implementación del aula invertida. El rango de búsqueda propuesta limita el recojo de mayor cantidad de artículos científicos. La elección del idioma es un limitante para recabar información más actualizada.

Este tipo de investigación sirve de base para futuras líneas de investigación como: indagar el efecto del aula invertida en los docentes y estudiantes de todos los niveles educativos, ampliar la revisión sistemática a los diversos beneficios que proporciona el aula invertida, considerando publicaciones en idioma inglés y con mayor rango de búsqueda en años.

\section{REFERENCIAS}

Aguayo, M., Bravo, M., Nocetti, A., Concha, L., \& Aburto, R. (2018). Perspectiva estudiantil del modelo pedagógico flipped classroom o aula invertida en el aprendizaje del Inglés como lengua extranjera. Revista Educación, 43(1), 97-112. https://doi.org/10.15517/revedu.v43i1.31529 
Barends, E., Rousseau, D., \& Rob, B. (2017). Guía CEMBa para TEMAS EVALUADOS CRÍTICAMENTE en Management y Organizaciones.

Basso, M., Bravo, M., Castro, A., \& Moraga, C. (2018). Propuesta de modelo tecnológico para Flipped Classroom (T-FliC) en educación superior. Revista Electronica Educare, 22(2). https://doi.org/10.15359/ree.22-2.2

Bergmann J., S. A. (2012). Flip Your Classroom: Reach Every Student in Every Class Every Day. En International Society for Technology in Education. www.cedro.org

Blasco, A., Lacruz, J., \& Sarsa, J. (2018). Percepción de los estudiantes al «invertir la clase» mediante el uso de redes sociales y sistemas de respuesta inmediata Students' perception of flipped classroom through the use of social networks and classroom response systems. Revista de Educación a Distancia, 57(6), 31-32. https://doi.org/10.6018/red/57/6

Campillo, F., Miralles, P., \& Sánchez, R. (2019). La enseñanza de ciencias sociales en educación primaria mediante el modelo de aula invertida. Revista Interuniversitaria de Formación del Profesorado, 94(33.3), 347-362. http://eds.b.ebscohost.com/eds/pdfviewer/pdfviewer?vid=7\&sid=62fa2388-38d7-4fa29290-ffb9b5bf28f7\%40sessionmgr103

Cuenca, P., García, S., Ferriz, A., \& Tortosa, J. (2021). Análisis comparativo de los perfiles motivacionales y el Estado de Flow entre una metodología tradicional y la metodología Flipped Classroom en estudiantes de Educación Física. Retos, 39(39), 338-344. https://doi.org/10.47197/retos.v0i39.78574

Díaz, V., \& Calzadilla, A. (2016). Artículos científicos, tipos de investigación y productividad científica en las Ciencias de la Salud. Revista Ciencias de la Salud, 14(1), 115-121. https://doi.org/10.12804/revsalud14.01.2016.10

Domínguez, F., \& Palomares, A. (2020). El aula invertida como metodología activa para fomentar la centralidad en el estudiante como protagonista de su aprendizaje. Contextos Educativos. Revista de Educación, 26(26), 261-275. https://doi.org/10.18172/con.4727

Espada, M., Rocu, P., Navia, J., \& Gómez, M. (2020). Rendimiento Académico y Satisfacción de los Estudiantes Universitarios hacia el Método Flipped Classroom. Profesorado, 24(1), 116135. https://doi.org/10.30827/profesorado.v24i1.8710

Fonseca, L. V. \& Melo, L. A. (2019). El uso del aula invertida como estrategia docente para la enseñanza de contenidos a través del inglés a niños en etapa inicial del colegio bilingüe Hispanoamericano Conde Ansúrez de Bogotá. En Maestría en Didáctica de las Lenguas. https://ciencia.lasalle.edu.co/maest_didactica_lenguas/3

Fúneme, C. (2019). El aula invertida y la construcción de conocimiento en matemáticas: el caso de las aplicaciones de la derivada - Dialnet. TED, 45, 159-174. https://dialnet.unirioja.es/servlet/articulo?codigo=6891185 
García, I., Higueras, L., \& Martínez, E. (2018). Hacia la implantación de comunidades profesionales de aprendizaje mediante un liderazgo distribuido: Una revisión sistemática. Revista Iberoamericana sobre Calidad, Eficacia y Cambio en Educación. https://doi.org/10.15366/reice2018.16.2.007

García, M., Porto, M., \& Hernández, F. (2019). El aula invertida con alumnos de primero de magisterio: fortalezas y debilidades. REDU. Revista de Docencia Universitaria, 17(2), 89106. https://doi.org/10.4995/redu.2019.11076

Garzon, A., \& Ibañez, J. (2020). Aula invertida, una estrategia que incide en el logro de aprendizaje. Repositorio de GRIAL. https://repositorio.grial.eu/handle/grial/2118

Gaviria, D., Arango, J., Valencia, A., \& Bran, L. (2019). Percepción de la estrategia aula invertida en escenarios universitarios. Revista Mexicana de Investigación Educativa, 24(81). https://www.comie.org.mx/revista/v2018/rmie/index.php/nrmie/article/view/1280/120 8

Gómez, L., \& Gómez, P. (2018). La experiencia de aprendizaje del estudiante de Teorías de la Comunicación en Periodismo desde el modelo de clase inversa. Estudios Sobre el Mensaje Periodistico, 24(2), 1321-1338. https://doi.org/10.5209/ESMP.62217

Guillén, F., Magaña, E., Rivas, E., \& del Río, R. (2020). Efectos sobre la metodología Flipped Classroom a través de Blackboard sobre las actitudes hacia la estadística de estudiantes del grado de educación primaria: Un estudio con Anova Mixto. Belo Horizonte, 13(3), 121139. https://doi.org/10.35699/1983-3652.2020.25107

Gutiérrez, J., \& Serrano, J. (2018). Tecnicas para el proceso de busqueda, acceso y seleccion de informacion digital: los operadores. Digital Education Review, 34. http://greav.ub.edu/der/

Gutierrez, L. (2012). Vista de Conectivismo como teorí-a de aprendizaje: conceptos, ideas, y posibles limitaciones. Educación y Tecnología, 1, 111-122. http://revistas.umce.cl/index.php/edytec/article/view/39/pdf

Hernández, C., Prada, R., \& Gamboa, S. (2020). Formación docente previa al servicio: escenarios activos desde un aula invertida una perspectiva. International Journal of Agriculture and Biology, 13(5), 213-222. https://doi.org/10.4067/S0718-50062020000500213

Hernandez, R., Fernandez, C., \& Baptista, M. (2014). Metodología de la investigación (S. A. D. C. . McGRAW-HILL / INTERAMERICANA EDITORES (ed.); Sexta). www.elosopanda.com\%7Cjamespoetrodriguez.com

Hinojo, F., Aznar, I., Romero, J., \& Marín, J. (2019). Influencia del aula invertida en el rendimiento académico. Una revisión sistemática. Campus Virtuales, 8(1), 9-18. www.revistacampusvirtuales.es 
Keengwe, J., \& Onchwari, G. (2015). Handbook of research on active learning and the flipped classroom model in the digital age. En Handbook of Research on Active Learning and the Flipped Classroom Model in the Digital Age. IGI Global. https://doi.org/10.4018/978-14666-9680-8

López, C. (2020). Práctica : Búsqueda bibliográfica en fuentes de información. Ejemplo en: Trip y PsycINFO. Brújula Repositorio Institucional. https://repositorio.uloyola.es/handle/20.500.12412/2207

López, J., Pozo, S., Fuentes, A., \& López, J. (2019). Creación de contenidos y flipped learning: un binomio necesario para la educación del nuevo milenio. Revista Espanola de Pedagogia, 77(274), 535-555. https://doi.org/10.22550/REP77-3-2019-07

Martínez, W., \& Esquivel, I. (2018). Uso del modelo de aprendizaje invertido en un bachillerato público Using the flipped learning model in a public high school. RED. Revista de Educación a Distancia. Núm, 58(58), 31-41. https://doi.org/10.6018/red/58/11

Medina, J., Calla, G., \& Romero, P. (2019). Las teorías de aprendizaje y su evolución adecuada a la necesidad de la conectividad. LEX, 17(23), 377. https://doi.org/10.21503/lex.v17i23.1683

Mendoza, G., \& Ledo, C. (2020). La mesa invertida de aprendizaje: una propuesta de trabajo grupal para el fortalecimiento de la metodología del aula invertida. Metodología del aula invertida para el trabajo grupal.Revista Didasc@lia: Didáctica y Educación, 11(5), 220-231.

Mengual, S., López, J., Fuentes, A., \& Pozo, S. (2020). Modelo Estructural de Factores Extrínsecos Influyentes en el Flipped Learning. Educacion XX1, 23(1), 75-101. https://doi.org/10.5944/educxx1.23840

Monroy, M., \& Monroy, C. (2019). El aula invertida versus método tradicional: En la calidad del aprendizaje. Ctes, 6(11), 1-17. file:///C:/Users/DELL/Downloads/692-2811-1-PB.pdf

Ojando, E., Simón, J., Prats, M., Martínez, M., Santaolalla, E., \& Torres, J. (2019). Evaluación de una experiencia formativa en Flipped Classroom para profesores universitarios de la Universidad Pontificia Comillas de Madrid. En Aloma: Revista de Psicologia, Ciències de l'Educació i de l'Esport (Vol. 37, Número 2). http://www.revistaaloma.net/index.php/aloma/article/view/384

Peña, S. (2020). La concepción del aprendizaje y la Evaluación en alumnos de Educación Primaria. Panorama, 14(27). https://doi.org/10.15765/pnrm.v14i27.1525

Prieto, A., Prieto, B., \& Begoña, P. (2016). Una experiencia de flipped classroom. CITIC-UGR Universidad de Granada, 237-244. http://atc.ugr.es/APrieto_videoclases.

Ruiz, M. y, \& Torres, A. (2020). Calidad de los aprendizajes de estudiantes de pedagogía: Influencia del modelo educativo. Revista Fuentes, 22(2), 238-250. 
https://doi.org/10.12795/revistafuentes.2020.v22.i2.01

Sánchez, C., \& Sánchez, T. (2020). El modelo flipped classroom, una forma de promover la autorregulación y la metacognición en el desarrollo de la educación estadística. Revista Interuniversitaria de Formación del Profesorado, 34(2), 121-142. https://doi.org/10.47553/rifop.v34i2.77713

Sánchez, E., Sánchez, J., \& Ruiz, J. (2019). Percepción del alumnado universitario respecto al modelo pedagógico de clase invertida. Magis, Revista Internacional de Investigación en Educación, 11(23), 151-168. https://doi.org/10.11144/Javeriana.m11-23.paur

Sánchez, J. (2018). Bases constructivistas para la integración de TICs. Revista Enfoques Educacionales, $6(1)$, 75-89. https://revistaderechoeconomico.uchile.cl/index.php/REE/article/view/48169

Sandí, J., \& Cruz, M. (2016). Propuesta Metodológica De Enseñanza Y Aprendizaje Para Innovar La Educación Superior. InterSedes, 17(36). https://doi.org/10.15517/isucr.v17i36.27100

Sepúlveda, M., \& Concepción, G. (2020). Técnicas e instrumentos de recolección de datos. Tecnológico Nacional de México. http://ri.utn.edu.mx/bitstream/handle/123456789/522/Técnicas e instrumentos de recolección de datos. pdf?sequence=1

Solano, E., Castellanos, S., López, M., \& Hernández, J. (2009). La bibliometría: una herramienta eficaz para evaluar la actividad científica postgraduada. Medisur: Revista Electrónica de las $\begin{array}{llll}\text { Ciencias Médicas en } & \text { 7(4), }\end{array}$ http://scielo.sld.cu/scielo.php?script=sci_arttext\&pid=S1727-897X2009000400011

Torrecilla, S., \& García, M. (2020). Flipped Classroom : estrategias de aprendizaje y rendimiento en ciencias. Edutec. Revista Electrónica De Tecnología Educativa, 72, 112-124. https://doi.org/10.21556/edutec.2020.72.1525

Urrútia, G., \& Bonfill, X. (2010). PRISMA declaration: A proposal to improve the publication of systematic reviews and meta-analyses. Medicina Clinica, 135(11), 507-511. https://doi.org/10.1016/j.medcli.2010.01.015

Veytia, M., Flores, L., \& Moreno, T. (2019). Clase invertida para el desarrollo de la competencia: uso de la tecnología en estudiantes de preparatoria. Revista Educación, 44(1), 30. https://doi.org/10.15517/revedu.v44i1.36961

Vigueras, J., \& Espinar, E. (2020). El aprendizaje experiencial y su impacto en la educación actual. Espinar, E. http://eds.b.ebscohost.com/eds/pdfviewer/pdfviewer?vid=15\&sid=46365d70-f8c34396-8c21-d2c2dc5fc5cf\%40pdc-v-sessmgr02 
Cabrera, S. Y., Rojas, E. M., Montenegro, D., y López, O.

\section{Para citar este artículo:}

Cabrera, S. Y., Rojas, E. M., Montenegro, D., y López, O. (2021). El aula invertida en el aprendizaje de los estudiantes: revisión sistemática. Edutec. Revista Electrónica De Tecnología Educativa, (77), 152-167. https://doi.org/10.21556/edutec.2021.77.1967 